\title{
INCIDENTAL ABNORMALITIES IN ORAL MUCOSAL CARCINOGENESIS OF v-H-ras TRANSGENIC MICE
}

\author{
Dewi Agustina \\ Oral Medicine Department, Faculty of Dentistry, Gadjah Mada University,
}

\begin{abstract}
The effect of individual oncogene on diverse cell types could be studied by using transgenic mice. The expression of transgene is mainly determined by the regulatory sequences chosen. Fifty four v-H-ras transgenic FVB/N mice and 54 parental FVB/N mice were used as the experimental and the control groups respectively. Each group was divided into nine subgroups with three different treatments i.e. 4-nitroquinoline 1-oxide (4NQO)-treated, phorbol 12,13-didecanoate (PDD)-treated and propane-1,2-diol (PD)-treated palatal of mice for 6, 16 or 24 weeks. Other four mice of parental mice were used as the untreated group. Two weeks after the last painting, all animals were sacrificed and the intra- or extra-oral tissues were removed and fixed in $4 \% \mathrm{~m} / \mathrm{v}$ paraformaldehyde for 24 hours. Hard tissue were then decalcified after the fixation was completed. Subsequently, standard procedure for H\&E staining was performed. The results of this study showed that 47 out of 54 transgenic mice produced spontaneous odontogenic, epidermal or mesenchymal neoplasms. After 24 weeks of painting with 4 NQO there was minimal evidence of palatal epithelial dysplasia in both transgenic and parental strain groups and neither the PDD nor PD groups showed evidence of dysplasia. From these results it was apparent that the effect of 4NQO and PDD was slower than reported for other strain of mice and that activated v-H-ras did not increase the rate of palatal mucosal carcinogenesis in the model used. On the other hand, incidental abnormalities were much detected especially in the experimental group.
\end{abstract}

Key words : incidental abnormalities, v-H-ras transgenic mice, oral mucosal carcinogenesis

\section{Introduction}

The creation of transgenic animals opens an exciting new era in oncology research. The potential for using specific promoters or enhancers to direct expression of heterologous genes to a specific cell type can generate various kinds of transgenic mice and as a consequence, the biological effects of an individual oncogene could be observed ${ }^{1}$. 
Besides that, the relationship between multistep oncogenesis and cooperativity of oncogenes and the effect of oncogenes on growth and differentiation could be studied more thoroughly ${ }^{2}$.

The neoplastic diseases developed in transgenic mice bearing H-ras genes under different regulatory elements are varied. It is necessary to note that tumours developed in the whey acidic protein (WAP)/mutated H-ras in response to lactogenic hormones. It can be seen that the expression of the transgene in the mammary glands and brains of lactating females was at a low level, but no expression could be detected after lactation terminated. Mammary tumours only developed in one founder female after 11 months, but the expression of WAP/ras RNA was increased. These results lead to an assumption that increased expression may have been due to an alteration in mRNA stability. From the description above a conclusion can be drawn that a transgenic mutant gene can elicit abnormal levels of expression in several different ways ${ }^{3}$.

Offspring heterozygous for both MMTV (mouse mammary tumor virus)/myc and MMTV/ras has been successfully generated. $50 \%$ of MMTV/myc female transgenic mice produced mammary tumours by 325 days of age compared with $50 \%$ of MMTV/ras female transgenic mice which developed tumours by 168 days of age. By contrast, using MMTV/ras/myc, the female transgenic mice could develop mammary tumours by 46 days of age, and by 163 days of age all female dual carriers had developed tumours. Furthermore, 21 dual carrier mice developed 35 proliferative disturbances, 9 animals each demonstrated two separate, coexistent processes, one dual carrier animal demonstrated three coexistent processes, whereas another demonstrated four associated proliferative processes (mammary carcinoma, malignant lymphoma, salivary gland neoplasm, and Harderian gland hyperplasia). These coexistent processes were not detected in either MMTV/myc or in MMTV/ras transgenic mice siblings ${ }^{4}$. From these results, it appears likely that the presence of a second oncogene is essential for the development of malignancy ${ }^{3}$.
Furthermore it seems that additional somatic events appear to be required for the onset of malignancy besides the presence of both oncogenes ${ }^{3,4}$. The results described above corroborate a view that particular cell types determine the expression of oncogenes and that this cell type may vary from tissue to tissue ${ }^{2}$.

The development of mammary tumour has been studied in various ways using transgenic mice. The findings inspired a study in oral mucosal carcinogenesis using v-H-ras transgenic mice and presumably had v-H-ras initiated cells. H-ras gene was chosen as an initiating gene since it is consistent with the previous findings that mutation of $\mathrm{H}$-ras gene in codons $12^{5}$ and $61{ }^{6}$ have been detected in murine and rat of oral squamous epithelia after induced by 4 NQO. It was evident that initiated cells produce isolated "latent tumour cells" that are not able to be distinguished morphologically from the surrounding non-neoplastic cells. The presence of these latent tumour cells can be demonstrated by a subsequent promoting agent, which leads to morphologically identifiable tumour cells ${ }^{7}$. The promoting agents have to be applied after the initiating agents ${ }^{8}$. Two promoters were used in this study i.e. 4nitroquinoline 1-oxide (4NQO) and phorbol 12, 13-didecanoate (PDD). These two agents have been much used to create oral cancer in animal models. The former can function also as an initiator.

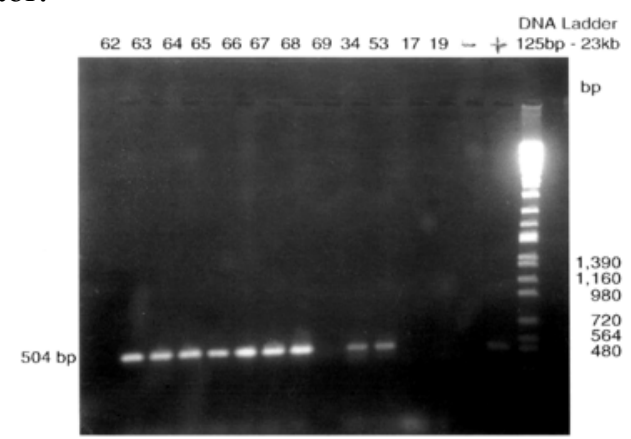

Fig. 1

Figure. 1. An example of the bands in a $\mathrm{PCR} / \mathrm{gel}$ analysis running at $504 \mathrm{bp}$ confirming the putative presence of the transgene in the experimental mice. For comparison three control mice (band number : $69,17,19)$ did not show the transgene. 


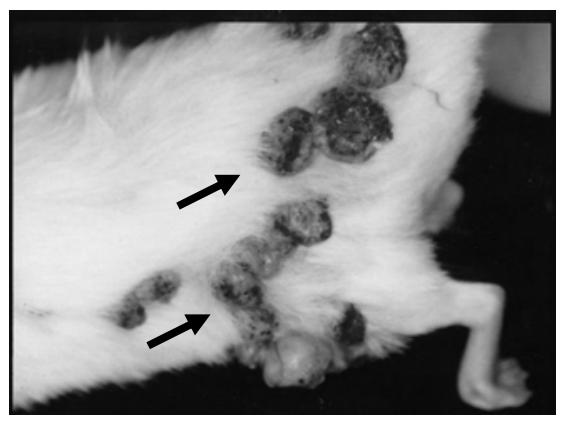

Figure. 2. A photograph illustrating multiple extraoral papillomas (arrow) which developed around the posterior extremities of a v-Hras transgenic mouse (X4).

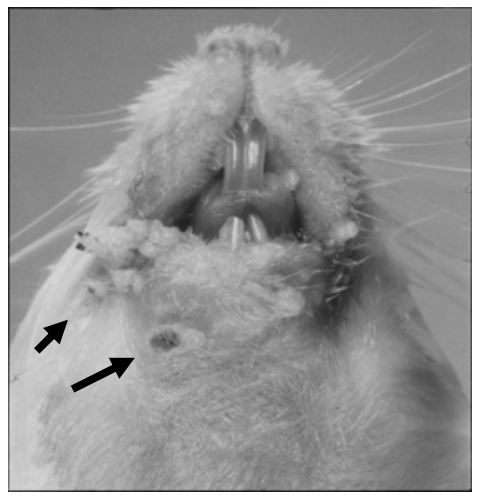

Figure. 3. A photograph of an example of the fractured lower incisors of a $30 \mathrm{wk}$-old v$\mathrm{H}$-ras transgenic mouse which were usually first noticed when the mice were $13-17$ wk of age. Note also the circumoral papillomas (arrow) (X5).

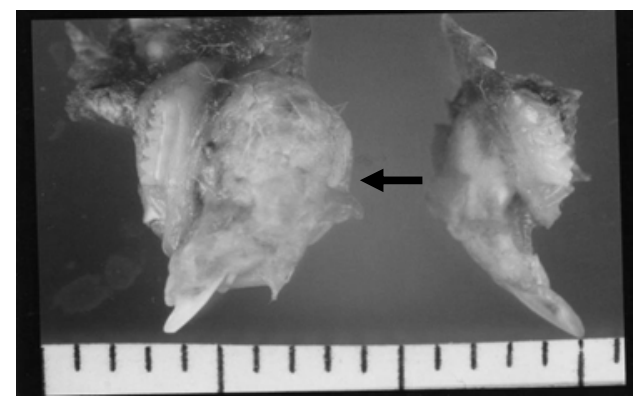

Figure. 4. A photograph showing an example of a mandibular enlargement which occurred in a v-H-ras transgenic mouse compared with the normal one. Enlargement of the body of the mandible (arrow) usually occurred on one side, appearing as a diffuse thickening which occasionally involved the face (X8).
The aim of this present study was to observe the abnormalities occurred in transgenic mice that bearing $\mathrm{v}-\mathrm{H}-\mathrm{ras}$ gene carried by $\zeta$ globin promoter after palatinally induced by promoting agents. Tumour promoters could act to enhance the outgrowth of initiated cells, as well as to 'activate' their program of differentiation ${ }^{9}$. Likewise, tumour promoters such as the phorbol esters, are capable of enhancing the phenotypic properties of neoplastic cells which are pre-existant, but lie dormant in the normal tissue. The significance of the present study should contribute to our understanding of the role of v-H-ras oncogene in oral mucosal carcinogenesis and in the development of other abnormalities.

\section{Materials and Methods}

In the present study 54 transgenic FVB/N mice (25 males, 29 females) (provided by the LICR, Melbourne, Victoria, Australia and having been supplied originally by Charles River Laboratory, UK) and 54 parental FVB/N mice (37 males, 17 females) (purchased from Garvan Institute of Medical Research, NSW, Australia) were used as the experimental and control groups respectively. Four mice ( 2 males, 2 females) of the parental strain were used as an untreated control group. The age of the mice used was between 11-17 wk. PCR analysis has been carried out to confirm the presence of the transgene in the experimental mice (Fig. 1). The parental strain $(\mathrm{FVB} / \mathrm{N})$ of mice were not subjected to PCR analysis. The transgenic mice used in the present study were offspring of the founder $\mathrm{v}$-H-ras transgenic mice. It was assumed that was no gender bias in terms of the response to the promoting agents. The animals were housed in groups according to gender and litter mate status in stainless steel and plastic cages at constant humidity and at a temperature of $20^{\circ} \mathrm{C}$ with 12 hours light in the Alumni Research Centre of the School of Dental Science (for the control and the untreated mice) and in the Animal House of LICR (for the experimental mice). They were fed irradiated mouse feed (Barastoc, Victoria, Australia) and had autoclaved water ad libitum. 
Table 1. Number and grouping of v-H-ras transgenic FVB/N (experimental) and of parental FVB/N (control) mice

\begin{tabular}{lcc}
\hline Group & Number of Experimental mice & Number of Control mice \\
\hline PD/6 wk & 6 & 6 \\
$\mathrm{PD} / 16 \mathrm{wk}$ & 6 & 6 \\
$\mathrm{PD} / 24 \mathrm{wk}$ & 6 & 6 \\
& & 6 \\
$\mathrm{PDD} / 6 \mathrm{wk}$ & 6 & 6 \\
$\mathrm{PDD} / 16 \mathrm{wk}$ & 6 & 6 \\
$\mathrm{PDD} / 24 \mathrm{wk}$ & 6 & 6 \\
& & 6 \\
$4 \mathrm{NQO} / 6 \mathrm{wk}$ & 6 & 6 \\
4NQO/16 wk & 6 & 4 \\
4NQO/24 wk & 6 & 58 \\
& & \\
Untreated & 54 & \\
\hline Total & & \\
\hline PD : propane-1,2-diol ; PDD : phorbol 12,13-didecanoate ; 4NQO : 4-nitroquinoline 1-oxide ; wk : week
\end{tabular}

Table 2. Incidental abnormalities in control (parental) mice

\begin{tabular}{|c|c|c|c|c|c|c|c|c|c|}
\hline \multirow[t]{2}{*}{ Abnormalities } & PD & PD & PD & PDD & PDD & PDD & $4 \mathrm{NQO}$ & $4 \mathrm{NQO}$ & $4 \mathrm{NQO}$ \\
\hline & $6 \mathrm{wk}$ & $16 \mathrm{wk}$ & $24 \mathrm{wk}$ & $6 \mathrm{wk}$ & $16 \mathrm{wk}$ & $24 \mathrm{wk}$ & $6 \mathrm{wk}$ & $16 \mathrm{wk}$ & $24 \mathrm{wk}$ \\
\hline Extra-oral papilloma & 0 & 0 & 0 & 0 & 0 & 0 & 0 & 0 & 0 \\
\hline Intra-oral papilloma & 0 & 0 & 0 & 0 & 0 & 0 & 0 & 0 & 2 \\
\hline Mandibular enlargement & 0 & 0 & 0 & 0 & 0 & 0 & 0 & 0 & 0 \\
\hline Alveolar enlargement & 0 & 0 & 0 & 0 & 0 & 0 & 0 & 0 & 0 \\
\hline Soft tissue growth & 0 & 0 & 1 & 0 & 0 & 0 & 0 & 1 & 0 \\
\hline Splenomegaly & 0 & 0 & 0 & 0 & 0 & 0 & 0 & 0 & 0 \\
\hline Tongue/FOM alteration & 0 & 0 & 0 & 0 & 0 & 0 & 0 & 0 & 0 \\
\hline Tooth fracture & 0 & 0 & 0 & 0 & 0 & 0 & 0 & 0 & 0 \\
\hline
\end{tabular}

Table 3. Incidental abnormalities in experimental (transgenic) mice

\begin{tabular}{|c|c|c|c|c|c|c|c|c|c|}
\hline \multirow[t]{2}{*}{ Abnormalities } & PD & PD & PD & PDD & PDD & PDD & 4NQO & 4NQO & $4 \mathrm{NQO}$ \\
\hline & $6 \mathrm{wk}$ & $16 \mathrm{wk}$ & 24 wk & $6 \mathrm{wk}$ & $16 \mathrm{wk}$ & $24 \mathrm{wk}$ & $6 w k$ & $16 \mathrm{wk}$ & $24 \mathrm{wk}$ \\
\hline Extra-oral papilloma & 0 & 2 & 6 & 0 & 5 & 6 & 1 & 1 & 6 \\
\hline Intra-oral papilloma & 0 & 0 & 0 & 0 & 0 & 0 & 0 & 1 & 2 \\
\hline Mandibular enlargement & 1 & 1 & 0 & 1 & 0 & 1 & 2 & 3 & 1 \\
\hline Alveolar enlargement & 0 & 1 & 1 & 2 & 6 & 2 & 0 & 2 & 1 \\
\hline Soft tissue growth & 0 & 1 & 0 & 0 & 0 & 0 & 2 & 1 & 1 \\
\hline Splenomegaly & 0 & 1 & 0 & 0 & 0 & 0 & 0 & 1 & 0 \\
\hline Tongue/FOM alteration & 0 & 0 & 0 & 0 & 0 & 0 & 0 & 0 & 1 \\
\hline Tooth fracture & 3 & 1 & 0 & 4 & 1 & 4 & 3 & 4 & 1 \\
\hline
\end{tabular}




\section{Ethics approval}

Approval was given for the present study by the Hospital Campus Animal Ethics Committee, Royal Melbourne Hospital, Parkville, Victoria (Approval No. 32/93) and the Animal Experimentation Ethics Committee of the University of Melbourne, Parkville, Victoria (Approval No. 230/025/0/93/1476).

\section{Materials}

Three chemical agents were used in this study i.e. 4-nitroquinoline 1-oxide (4NQO) (Sigma Chemical Company, St. Louis, USA), phorbol 12, 13-didecanoate (PDD) (SigmaAldrich Pty. Ltd., Castle Hill, Australia) and propane-1,2-diol (PD) (BDH Limited, Poole, England). 4NQO was prepared at a $0.5 \% \mathrm{~m} / \mathrm{v}$ concentration in PD and stored at $5^{\circ} \mathrm{C}$ between applications. PDD was prepared at a $0.1 \% \mathrm{~m} / \mathrm{v}$ solution in PD. Besides being used as a solvent for $4 \mathrm{NQO}$ and PDD, PD was used as a vehicle in this study.

\section{Technique of application of the chemical agents}

The three chemical agents described above were applied with a single brush stroke to the palate of the mice from the junction of the hard and soft palates to the incisive papilla. A No. 2 artist's brush which delivered a relatively constant quantity $(0.15 \pm 0.03 \mathrm{mg})$ of the test solution was used for application. No anaesthetic agent was used in this procedure. The application was carried out thrice-weekly for 6,16 or 24 week periods.

\section{Grouping of animals}

Both the experimental and the control goup was divided into nine subgroups with three different treatments i.e. 4NQO-treated, PDD-treated and PD-treated palatal of mice for 6,16 or 24 weeks. Four mice of parental mice were used as the untreated group (Table 1).

\section{Tissue sampling}

Two weeks after the last painting, the mice were killed by cervical dislocation in which aether anaestheticum was given previously. Following macroscopic examination gross pathological tissues either intra- or extraoral were removed for microscopic assessment.

Histological processing and staining of tissues All specimens were fixed in $4 \% \mathrm{~m} / \mathrm{v}$ paraformaldehyde for 24 hours. Processing of hard tissues (bone and teeth) involved decalcification in Neutral EDTA pH 7.0 after fixation was completed. After fixation and decalcification was completed, the specimens were transferred into $0.5 \mathrm{M}$ sucrose in $0.1 \mathrm{M}$ Phosphate Buffer for a minimum of 24 hours. Then, standard procedure for Haematoxylin and Eosin (H\&E) stained section was performed.

\section{Macroscopic and microscopic assessment}

Macroscopic examination was used to describe anatomic location, consistency, size and colour of the pathologic tissues. Microscopic examination was performed by using a light microscope for the assessment of H\&E stained sections. Microscopic assessments of epithelial dysplasia in palatal mucosa used a modification of the semi-quantitative method of grading of epithelial atypia described by Smith and Pindborg ${ }^{10}$.

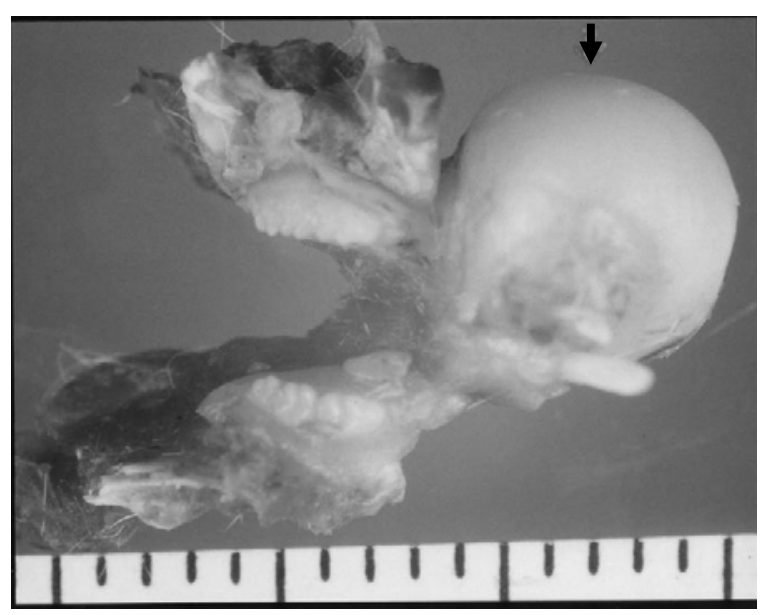

Figure. 5. A photograph demonstrating an example of an alveolar enlargement (arrow) which occurred in a v-H-ras transgenic mouse. Enlargement of the alveolar ridge usually occurred on the lingual of one or two teeth as a prominent firm mass (X8). 


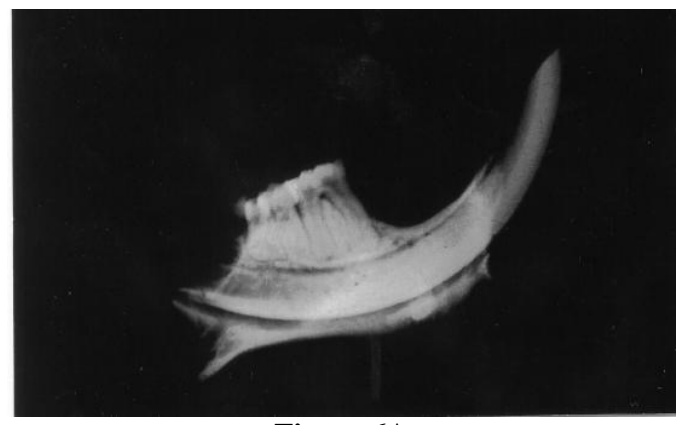

Figure 6A

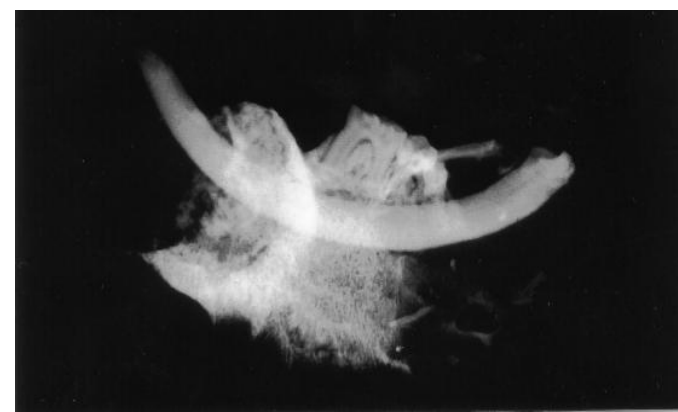

Figure 6B

Figure. 6. The corresponding radiograph of the mandibular enlargement (B) compared with that of the normal mandible (A). The radiographic appearance of the mandible showed a considerable expansion of the bone with a multilocular appearance in a 40 wk-old v-H-ras transgenic mouse. Note the obliteration of the pulp in the incisor in B. This tooth had fractured.

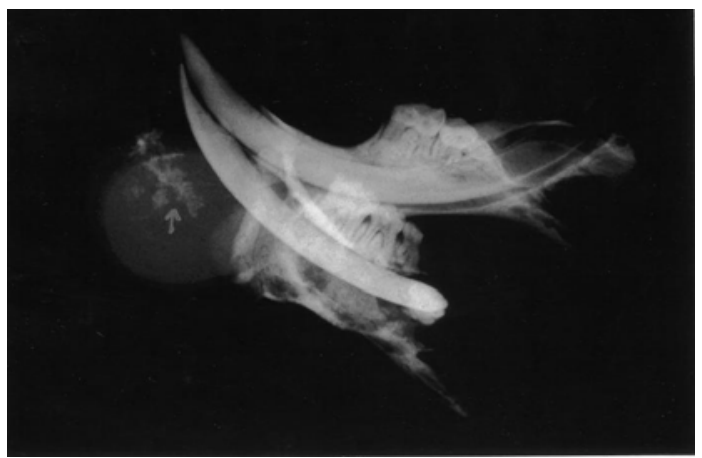

Figure. 7. The corresponding radiograph of the alveolar enlargement in a 48 wk-old v-Hras transgenic mouse. Expansion of the alveolar ridge contained a fine trabecular arrangement (arrow). Note the obliteration of the pulp of the incisor on the side of the jaw affected by a tumour.

\section{Results}

According to the macroscopic appearance, there were no observable differences between that of the palatal mucosa of the experimental and control mice related to the solutions or time periods used. The microscopic assessment of the palatal mucosa showed that the dysplastic changes only occurred in the mice treated with $4 \mathrm{NQO}$ either in the experimental or in the control group. Only a small fraction (6 out of 106) of the mice treated with this substance was shown to have epithelial dysplasia, mostly graded as mild. Only two 4NQO-treated mice showed moderate dysplasia.

Incidental abnormalities occurred in 47 of the 54 mice in the experimental group and 4 of the 54 mice in the control group. In the experimental group, there were 21 mice with a single abnormalities, 16 mice with two distinct abnormalities, 9 mice with three distinct abnormalities and one mouse of 16-wk-PD treated group had four distinct abnormalities (Tables 2, 3).

The most characteristic pathological changes observed in the experimental mice used in the present study were cutaneous papillomas. The occurrence of extra-oral papillomas usually commenced at approximately $14-26$ wk of age. These papillomas enlarged with increasing time. They were well-circumscribed exophytic growth with a diameter from $0.5 \mathrm{~mm}$ to $10 \mathrm{~mm}$, and a length of from $2 \mathrm{~mm}$ to $5 \mathrm{~mm}$. Microscopic examination showed that both intra- and extra papillomas were similar and were typical of classical papillomas. The papillomas usually developed on the surface of the skin of the face (around the mouth), anterior and posterior extremities, ventral and dorsal sites especially on the back and around the anus (Figs. 2, 3). The papillomas were either single and of a larger size, or they were in groups of small papillomas, such as occurred around the mouth and anus.

The enlargements of the body of the mandible and of the alveolar ridge could not always be differentiated, since the alveolar ridge enlargements occasionally merged with a larger 
swelling of the body of the mandible (Fig. 4, 5). These enlargements were firstly noticed at approximately $17-24$ wk of age. The microscopic features of the mandibular enlargements and of the alveolar enlargements were similar. There were two characteristic histological patterns that could be identified as an aggressive epithelial tumour and a malignant mesenchymomas. The radiographic appearance of the mandible showed a considerable expansion of the bone with a multilocular appearance in 40 wk-old $\mathrm{v}-\mathrm{H}$-ras transgenic mouse (Fig. 6B) compared with that of the normal mandible (Fig. 6A) Expansion of the alveolar ridge contained a fine trabecular arrangement (Fig. 7).

Soft tissue growths were detected at several sites. These abnormalities were firstly noticed at approximately $25 \mathrm{wk}$ of age. The growth which occurred in the PD-treated group was found within the right parotid gland and had spread to involve the cheek mucosa. Histological examination showed this growth to be a typical squamous cell carcinoma (SCC). While four growths in 4NQO-treated mice were localized i.e.. one occurred as a round brownish lump attached to the posterior surface of the spleen and was identified as a lymphoma ; two growths were found in chest cavity ; the other was an ovoid growth of firm consistency (7X6X5 mm) arising in animal, under the skin between the left eye and the left ear. This latter swelling was identified histologically as a SCC. One of the growths found in chest cavity was of firm consistency, creamy in color measuring $14 \mathrm{X} 10 \mathrm{~mm}$ with a diagnosis of SCC. Another growth with a rectangular shape, brownish/reddish in color measuring 21X13X5 $\mathrm{mm}$ in an animal from the 4NQO/16 wk group was diagnosed as an atypical thymic lymphoma. There were two soft tissue swellings found in the control group. One was found in an animal from the $\mathrm{PD} / 24$ wk group within the posterior third of the tongue on the right side and comprised a rectangular firm swelling, yellowish in color and measuring $4 \times 2 \mathrm{~mm}$ that diagnosed as an typical epidermoid cyst. The other swelling in an animal from the 4NQO/16 wk group and occurred in the upper labial sulcus on the left side. It was cream-coloured, fragile swelling, irregular in shape with ulceration on the oral cavity surface and diagnosed as a SCC.

Fractures of the lower incisor teeth were noticed when the experimental mice were 13 17 wk of age. Incisor tooth fractures occurred in 4 out of 18 mice of PD-treated group, 9 out of 18 mice of PDD-treated group and 8 out of 18 mice of 4NQO-treated group. The teeth fractured at approximately one-third to one-half of the length of the clinical crown of the lower incisors. In most animals with fractured incisors, it was one lower incisor tooth that had fractured (Fig. 3).

The splenomegaly occurred in two of the experimental mice was diagnosed as hyperplasia of the spleen. One experimental mouse from the 4NQO-treated group had extensive ulceration of floor of mouth on left side. This lesion was identified as a SCC with inverted papillary configuration.

\section{Discussion}

By comparison with the results of the previous studies, the results of these studies can be attributed in part to strain differences in the process of carcinogenesis. Hence it is obviously important to consider the strain of animal when designing and interpreting carcinogenesis experiments.

It was noted that during the course of the experiment the condition of the experimental mice deteriorated. It was attributed to the incidental abnormalities that were noted more frequently in the experimental mice than in the control mice. The incidental abnormalities that occurred in the experimental mice were similar to those described by previous researchers who used v-H-ras transgenic mice promoted with the embryonic $\zeta$-globin gene. They found that $20 \%$ of the animals $(n=95)$ showed spontaneous neoplasms with an average latency of $36 \mathrm{wk}^{11}$. The occurrence of the incidental abnormalities in the present study was approximately three months earlier. The tumours such as unusual ameloblastomas and mesenchymomas of the 
jaw, SCC and lymphomas were described by previous researchers and were found also in the present study. The mandibular tumours described by previous researchers were ameloblastomas, invasive SCC, odontogenic myxoma and occasionally the swellings were caused by cysts lined by a squamous epithelium ${ }^{11}$.

The spontaneous nature of the incidental pathological conditions in the experimental (transgenic) mice has no ready explanation, but as previous study suggested they may occur because of the induction of ras or because of "the clonal expansion of a rare subpopulation of ras-expressing cells" ${ }^{12}$. Concerning the method of producing transgenic animals by DNA microinjection, it was clear that the position in the chromosome of the fused oncogene cannot at present be determined. The difference in the results of the present study and those of the other investigations in which similar transgenic mice were used are most likely the result of different insertions of the oncogene.

The abnormalities found in the present study that have not been reported following previous investigations were associated with fractures of lower incisor teeth. This abnormality was not recorded in the control mice and it is likely that it was attributable to the v-H-ras transgene. Since not all of the experimental mice in the present study developed this abnormality (21 out of 54 mice), the findings could be attributed to the random chromosomal position into which the injected DNA was located. Although all mice carried the same oncogene, different insertions of the same transgene could dramatically affect the different pattern of abnormalities depending on the distribution of the oncogene expression ${ }^{13,14}$. The pattern of tooth fractures i.e. mainly of a lower continuously growing incisor tooth, was difficult to explain. The fractured teeth had some of the histological features of dentinal dysplasia Type $\mathrm{I}^{15}$ and a discoloured incisor tooth was noted which is an occasional feature of dentinal dysplasia in humans. It is likely that the fractures were related to a reduction in the physical strength of the dentine. Dentinal dysplasia in humans is of unknown aetiology and is an autosomal dominant with $100 \%$ penetration. The findings from the present study may provide an animal model for the study of this disorder.

Apart from the possibility of a v-H-ras associated dentine defect it was also noted that invasive neoplasms caused resorption of teeth which could have been another cause of incisor teeth fracturing.

When compared with the tumours that developed in other transgenic mice bearing H-ras genes, but driven by different promoters, the types of tumours developed in the present study were different. When c-H-ras with elastase promoter was introduced, the development of pancreatic neoplasms were recorded. However when v-Hras/MMTV was integrated into the embryo of the mice no pancreatic tumours were found. The transgene of v-H-ras/MMTV was detected in mammary gland, salivary gland, spleen, thymus, lung, seminal vesicle and Harderian gland ${ }^{11}$. These results confirmed the suggestion that transgenic mice with either a different transgene or a different promoter produce a different range of abnormalities.

The results of the present study were similar to those of previous investigators who described multiple abnormalities in their transgenic mice with dual oncogenes (ras and myc) driven by MMTV, however with a single oncogene they did not find any coexistent abnormalities ${ }^{4}$. The occurrence of multiple incidental abnormalities noted in the experimental mice in the present study suggested that there were more oncogenes active than the v$\mathrm{H}-$ ras transgene. This suggestion needs to be confirmed by assaying for oncogenes in the tumour tissue. From the results of the present study and from the results of previous studies, squamous papillomas, lymphomas and splenomegaly were identified as the tumours produced by the v-H-ras transgenic mice promoted by $\zeta$ - globin gene.

\section{Acknowledgements}

I would like to thank to Professors PC Reade and AW Burgess for their guidance and suggestions during conducting this study. To AIDAB for providing the research funding and to 
the School of Dental Science, University of Melbourne and the Ludwig Institute for Cancer Research of Victoria, Australia for fascilities in doing animal experimentation and completion of this research.

\section{References}

1. Groner B, Schonenberger C, Andres A. Targeted expression of the ras and myc oncogenes in transgenic mice. Trends Genet. $1987 ; 3$ : 306308.

2. Jaenisch R. Transgenic Animals. Science 1988 ; $240: 1468-1474$.

3. Cory S, Adams JM. Transgenic mice and oncogenesis. Ann. Rev. Immunol. 1988; 6 : 2548.

4. Pattengale PK, Stewart TA, Leder A, Sinn E, Muller W, Tepler I, Schmidt E, Leder P. Animal Models of Human Disease. Am. .J of Pathology $1989 ; 135: 39-61$.

5. Yuan B, Heniford BW, Ackermann DM, Hawkins BL, Hendler FJ. Harvey ras (H-ras) point mutations are induced by $4 \mathrm{NQO}$ in murine oral squamous epithelia, while squamous cell carcinomas and loss of heterozygosity occur without additional exposure. Cancer Res. 1994 ; $54: 5310-5317$.

6. Suzui M, Yoshimi N, Tanaka T, Mori H. Infrequent Ha-ras mutations and absence of $\mathrm{Ki}$ ras, N-ras and p53 mutations in 4NQO-induced rat oral lesions. Mol. Carcinog. 1995 ; 14 : 294298.
7. Berenblum I, Shubik P. An Experimental Study of the Initiating Stage of Carcinogenesis, and a Re-examination of the Somatic Cell Mutation Theory of Cancer. Br. J. Cancer 1949 ; 3 : 109118.

8. Miller EC, Miller JA. Mechanism of Chemical Carcinogenesis. Cancer 1981 ; 47 : 1055-1064.

9. Weinstein IB. Molecular Mechanism of Multistage Carcinogenesis. In : Nicolini eds. Chemical Carcinogenesis. New York : Plenum Press. 1982: 261-278.

10. Smith C, Pindborg JJ. Histological Grading of Oral Epithelial Atypia bu the Use of Photographic Standards. World Health Organization's International Reference Centre for Oral Precancerous Conditions. 1969 : 5-35.

11. Cardiff RD, Leder A, Kuo A. Multiple Tumours Types Appear in a Transgenic Mouse with the ras Oncogene. Am. J. of Pathol. 1993 ; 142 : 1199-1207.

12. Leder A, Kuo A, Cardiff RD. v-Ha-ras transgene abrogates the initiation step in mouse skin tumorigenesis : Effects of phorbol esters and retinoic acid. Proc. Natl. Acad. Sci. USA $1990 ; 87: 9178-9182$.

13. Adams TE, Alpert S, Hanahan D. Non-tolerance and autoantibodies to a transgenic self antigen expressed in pancreatic $\beta$ cells. Nature 1987 ; 325: 223-228.

14. Hanahan D. Dissecting Multistep Tumorigenesis in Transgenic Mice. Annu. Rev. Genet. 1988 ; $22: 479-519$.

15. Steidler NE, Radden BG, Reade PC. Dentinal dysplasia : A Clinicopathological Study of Eight Cases and Review of the Literature. British J. of Oral Maxillofacial Surg. $1984 ; 22$ : 274-286. 